\title{
On the robustness of elastic thickness estimates obtained using the coherence method
}

\author{
Ana Macario \\ Lamont-Doherty Earth Observatory, Palisades, New York \\ Alberto Malinverno \\ Schlumberger-Doll Research, Ridgefield, Connecticut \\ William F. Haxby \\ Lamont-Doherty Earth Observatory, Palisades, New York
}

\begin{abstract}
In order to uniquely determine the elastic thickness of the lithosphere, $T_{e}$, from gravity and topography data, the coherence method explicitly assumes that surface and subsurface loads are statistically uncorrelated. In many realistic cases (e.g., mountain building) this assumption is likely to be violated. We present the results of Monte Carlo simulations designed to measure the bias and variance of $T_{e}$ estimates when the degree of correlation, $R$, between fractal surface and subsurface loads is not null. In this study, $R$ denotes the linear correlation coefficient (Pearson's coefficient), and surface and subsurface loads are self-affine (fractal). According to our results, when there is no correlation between the loads $(R=0)$, the estimated $T_{e}$ is in excellent agreement with the simulated $T_{e}$ although its accuracy decreases for increasing plate stiffness. We also find that there is a strong likelihood of an upward bias in $\widehat{T}_{e}$ estimates if the dimensions of the study area are not adequate to fully resolve the longest wavelengths. This might partially explain why $T_{e}$ values for previous continental studies obtained using the coherence method are often higher than $T_{e}$ values obtained using conventional forward modeling techniques. As the degree of correlation increases, we observe a clear downward bias in the estimated $\widehat{T}_{e}$. When surface and subsurface loads are correlated by only a small amount, the fit between the computer-simulated and predicted coherences is still good for the longest wavelengths within the diagnostic wavebands. In light of these limitations, we use the coherence method to reassess $T_{e}$ estimates for the Alps. Our results indicate that the flexural behavior of the lithosphere to loading does not change significantly along the fold/thrust belt. The similarity between our $T_{e}$ results for the western $(30-39 \mathrm{~km})$ and eastern Alps (33-40 km) does not support the hypothesis that $T_{e}$ and the horizontal radius of curvature of mountain belts are correlated.
\end{abstract}

\section{Introduction}

The relationship between gravity and topography has been widely used to understand the mechanical behavior of the lithosphere to loads applied over geologic timescales (greater than $10^{4}$ years). The idea that the lithosphere behaves as an elastic thin plate overlying a weak fluid has been extensively tested, particularly over oceanic areas [e.g., Walcott, 1970; Watts and Cochran, 1974; Watts and Talwani, 1974; McNutt and Menard, 1982, Watts et al., 1980]. The most important result of these studies is that as the lithosphere gets older its mechanical strength (i.e., effective elastic thickness, $T_{0}$ ) increases as predicted by the plate-cooling model. The Airy local isostasy model represents an end-member in which the lithosphere cannot support any vertical shear stresses, that is, $T_{0}$ is null. In this case compensation occurs directly beneath the topography by thickening of a constant density crust.

Forsyth [1985] demonstrated that $T_{0}$ estimates obtained from conventional admittance techniques (the linear transfer

Copyright 1995 by the American Geophysical Union.

Paper number $95 \mathrm{JB} 00980$.

0148-0227/95/95JB-00980\$05.00 function between topography and gravity) are bound to be biased toward low values if the effects of subsurface density/mass variations and associated compensation are not taken into account [Dorman and Lewis, 1970; Lewis and Dorman, 1970; McKenzie and Bowin, 1976; Banks et al., 1977; McNutt and Parker, 1978; McNutt, 1980, 1983; Louden, 1981; Ribe and Watts, 1983]. These subsurface loads in the form of mafic intrusions, accreted lower crustal material, thermal anomalies, and compositional variations may represent an important mechanism for loading the lithosphere in addition to surface loads. Forsyth [1985] also showed that when admittance techniques are used in continent-wide studies [e.g., Lewis and Dorman, 1970; Banks et al., 1977; McNutt and Parker, 1978], the different provinces are, on average, weighted by the square of the amplitude of the their topographic relief. Thus, areas with small topographic expression related to high rigidities (e.g., interior cratons) are automatically underweighted.

A new method (hereinafter called the coherence method) for determining the flexural rigidity of the lithosphere when both surface and subsurface loads are present was proposed by Forsyth [1985]. The coherence method is based on the wavelength dependence of the coherence between the 
topography and Bouguer gravity and has been applied to a variety of continental [e.g., Bechtel et al., 1987; McNutt et al., 1988; Ebinger et al., 1989; Zuber et al., 1989; Bechtel et al., 1990] and oceanic [e.g., Blackman and Forsyth, 1991] tectonic settings. In order to uniquely determine $T_{e}$, the coherence method explicitly assumes that surface and subsurface loads are statistically uncorrelated. This crucial assumption is likely to be violated in many realistic cases. For example, while the Hawaiian-Emperor islands act as significant surface loads, seismic data suggests that subcrustal loads in the form of sill complexes present directly beneath the island are also loading the lithosphere [Watts et al., 1985]. In continental areas, subsurface loads may be present in the form of lithospheric wedges beneath fold/thrust belts [e.g., Nicolas et al., 1990].

In both cases cited above, one would expect the distribution of the surface loads to be spatially correlated to the distribution of subsurface loads. It is precisely the effect of load correlation on $T_{e}$ estimates obtained using the coherence method that we investigate in this study. Specifically, we present the results of Monte Carlo computer simulations designed to quantify the bias and variance in $T_{e}$ estimates when the degree of correlation between surface and subsurface loads is not null. First, we create synthetic fractal surface and subsurface loads with varying degrees of correlation. After calculating the deflection and gravity anomaly associated with the compensated loads, we use the coherence method to predict the best fitting $T_{0}$. Finally, the coherence method is used to reassess $T_{0}$ estimates for the Alps in light of the results/limitations learned from the Monte Carlo simulations.

\section{Formulation Summary}

We start by stating that, for an homogeneous thin elastic plate, the flexural rigidity of the plate, D, can be directly related to its thermally controlled effective elastic thickness, $T_{e}$, through Poisson's ratio, $\sigma$, Young modulus, E, and, characteristic flexural wavelength, $\lambda$ (see Table 1) [Turcotte and Schubert, 1982]:

$$
\begin{aligned}
& \mathrm{D}=\frac{\mathrm{E} \mathrm{T}_{0}^{3}}{12\left(1-\sigma^{3}\right)} \\
& \lambda=\pi\left(\frac{4 \mathrm{D}}{\Delta \mathrm{P}_{\mathrm{g}}}\right)^{1 / 4}
\end{aligned}
$$

where $\rho_{r}$ is the density contrast at the interface which provides compensation to the applied load, and $g$ is the gravitational acceleration.

Table 1. List of Physical Parameters

\begin{tabular}{lll}
\hline Parameter & \multicolumn{1}{c}{ Definition } & \multicolumn{1}{c}{ Value } \\
\hline E & Young modulus & $1.0 \times 10^{11} \mathrm{~N} \mathrm{~m}^{.2}$ \\
$\sigma$ & Poisson's ratio & 0.25 \\
$\Gamma$ & Grav. constant & $6.67 \times 10^{-11} \mathrm{~m}^{3} \mathrm{~kg}^{-1} \mathrm{~s}^{2}$ \\
$\mathrm{~g}$ & Grav. acceleration & $9.8 \mathrm{~m} \mathrm{~s}^{-2}$ \\
$\rho_{\mathrm{c}}$ & Custal density & $2800 \mathrm{~km}^{-3}$ \\
$\rho_{\mathrm{m}}$ & Mantle density & $3300 \mathrm{~kg} \mathrm{~m}^{-3}$ \\
& & \\
\hline
\end{tabular}

Using uppercase letters for topography $H(k)$ and Bouguer gravity $B(k)$ in the wavenumber domain, the coherence between gravity and topography is defined as [McKenzie and Bowin, 1976]

$$
\gamma_{0}^{2}(\bar{k})=\frac{\langle B(k) H(k)\rangle^{2}}{\langle H(k) H(k)\rangle\langle B(k) B(k)\rangle}
$$

where $k$ ( $2 \pi /$ wavelength) is the two-dimensional wavenumber vector, $\bar{k}$ is the average $\mathbf{k}$ for a wavenumber band; the asterisk indicates complex conjugation, and the angle brackets indicate averaging over discrete wavebands.

Coherence estimates obtained as described above may be positively biased by noise [Munk and Cartwright, 1966]. Unbiased coherence estimates are given by

$$
\gamma^{2}(\overline{\mathbf{k}})=\frac{\mathrm{n}(\overline{\mathbf{k}}) \gamma_{0}(\overline{\mathbf{k}})^{2}-1}{\mathrm{n}(\overline{\mathbf{k}})-1}
$$

where $n(k)$ is the number of independent Fourier coefficients within each discrete waveband.

In this study we adopt a simple two-layer density model in which the amplitude of the relief on the surface $H(k)$ (air-crust interface) and subsurface W(k) (crust-mantle) is given by

$$
\mathrm{H}=\mathrm{H}_{\mathrm{T}}+\mathrm{H}_{\mathrm{B}} \text { and } \mathrm{W}=\mathrm{W}_{\mathrm{T}}+\mathrm{W}_{\mathrm{B}}
$$

where $H_{T}$ and $H_{B}$ represent the amplitude of the surface relief due to loading on top and bottom, respectively; $W_{T}$ and $W_{B}$ represent the amplitude of the subsurface relief due to loading on top and bottom, respectively.

Consider the two-dimensional Fourier transform of the thin elastic plate equation [Banks et al., 1977]

$$
D k^{4} U(k)+P_{m} g U(k)=Q(k)
$$

where $\rho_{m}$ is the mantle density, $Q(k)$ is the applied load, and, $\mathrm{U}(\mathrm{k})$ is the amplitude of the plate deflection. By separating the components of surface and subsurface relief, Forsyth [1985] derived expressions for each component of relief at the surface $H(k)$ and Moho $W(k)$. The power of topography and gravity and cross-spectrum can be expressed in terms of the calculated relief components $\mathrm{H}_{\mathrm{T}}, \mathrm{H}_{\mathrm{B}}, \mathrm{W}_{\mathrm{T}}$, and $\mathrm{W}_{\mathrm{B}}$ used to obtain the predicted coherence as shown in (3). Assuming that the loads are uncorrelated (i.e., cross terms are left out), Forsyth [1985] derived the following expression for the predicted coherence:

$$
-\gamma_{0}^{2}(\bar{k})=\frac{\left\langle H_{T}(k) W_{T}(k)+H_{B}(k) W_{B}(k)\right\rangle^{2}}{\left\langle H_{T}^{2}(k)+H_{B}^{2}(k)\right\rangle^{2}\left\langle W_{T}^{2}(k)+W_{B}^{2}(k)\right\rangle^{2}}
$$

\section{Monte Carlo Simulation}

As stated above, the formulation for the predicted coherence explicitly assumes that surface and subsurface loads are statistically uncorrelated. In order to quantify the bias and variance in $T_{0}$ estimates, we designed Monte Carlo computer simulations for varying $T_{\text {o }}$ and degree of correlation, $R$, 
between surface and subsurface loads. In order to facilitate the discussion, the calculations performed in this study are divided into a forward and inverse part.

The forward part consists of creating the loads and calculating the gravity effect of the deflected plate. Based on the observation that fractal (power-law) statistics are a good approximation to continental topography [e.g., Mandelbrot, 1982; Mark and Aroson, 1984], surface and subsurface synthetic loads with a fractal geometry are used. Specifically, we use the mid-point displacement method [Peitgen and Saupe, 1988] to generate two-dimensional fractal Brownian motion surfaces representing the initial surface and subsurface loads. A fractal dimension of 2.5 for both surface and subsurface loads, compatible with values obtained for actual topography, is assumed. Initial tests using loads with fractal dimensions in the range of 2.3-2.7 showed that the estimated elastic thickness $\widehat{T}_{c}$ is not sensitive to the fractal dimension used. The load grids consist of $128 \times 128$ points regularly spaced at $8 \mathrm{~km}\left(10^{\circ} \times 10^{\circ}\right)$, and each simulation involves generating 100 synthetic pairs of such loads. Each set is linearly detrended, standardized (unit variance) and rescaled such that the load applied at the top of the plate equals the load applied at the bottom of the plate (coherence estimates are not very sensitive to the choice of the load ratio [Forsyth , 1985]). The loads are then emplaced on a thin elastic plate of thickness $T_{0}$ (we show the results for $T_{e}=20,40$, and $80 \mathrm{~km}$ ) and the gravity anomaly associated with the deflected crust-mantle interface is calculated using Parker's [1972] formulation. The Bouguer gravity, together with the final surface relief $H(k)$, is used to obtain the simulated coherence (equation (3)).

In the inverse part, the subsurface relief $W(k)$ is obtained by downward continuation of the Bouguer gravity anomaly. We then make an initial guess for $T_{b}$ and use the equations derived by Forsyth [1985] to solve for the different load components $\left(\mathrm{H}_{\mathrm{T}}, \mathrm{H}_{\mathrm{B}}, \mathrm{W}_{\mathrm{T}}\right.$, and $\left.\mathrm{W}_{\mathrm{B}}\right)$ and to obtain the predicted coherence estimates (equation (7)). In practice, we use an iterative process which consists of making two initial guesses for $T_{e}$ $\left(T_{e}=1\right.$ and $\left.150 \mathrm{~km}\right)$ and using an optimization procedure based on the golden-section search method [Press et al., 1991] to find $\widehat{T}_{e}$. The estimated $\widehat{T}_{e}$ is defined as the $T_{e}$ value that yields the smallest summed squared error between the simulated $g$ (equation 3) and predicted coherence $\gamma_{p}$ (equation (7)):

$$
E=\sum_{i=1}^{b}\left[\gamma^{2}(k)-\gamma_{p}^{2}(k)\right]^{2}
$$

where $b$ is the total number of equally spaced bands in the wavenumber domain. The mean estimated $\hat{T}_{e}$ value, $\left\langle\hat{T}_{e}\right\rangle$, is obtained by averaging over 100 independent estimates of $\widehat{T}_{e}$ (each single model run yields a $\widehat{T}_{e}$ estimate).

In order to evaluate the sensitivity of the $\widehat{T}_{e}$ estimates to the correlation between surface and subsurface loads, we assign a fixed amount of correlation $(R=-0.2,-0.4,-0.6$, and -0.8 at all wavelengths) between the fractal surface and subsurface loads. The degree of correlation is assigned in the space domain by starting with two standardized uncorrelated fractal surfaces, $s_{1}$ and $s_{2}$, and creating a third surface $s_{3}$ related to $s_{1}$ by $R$. The initial subsurface load, $s_{3}$, is related to the initial surface loads, $s_{1}$ and $s_{2}$ by

$$
s_{3}=R s_{1}+\left(\sqrt{1-R^{2}}\right) s_{2}
$$

The degree of correlation $R$ is intentionally chosen to be negative in order to impose mirror symmetry between surface and subsurface loads. In the context of this study, the degree of correlation $R$ denotes the linear correlation coefficient (i.e., Pearson's coefficient) given by the ratio of the covariance between $s_{1}$ and $s_{3}$ and their respective standard deviations (see the appendix). As both surfaces $s_{1}$ and $s_{2}$ are linearly detrended and standardized prior to assigning the degree of correlation $R$, it can be shown that, even after re-scaling constants are applied, $\mathbf{R}$ remains analytically equivalent to Pearson's linear correlation coefficient (see appendix).

It is important to note that the number of wavenumber bands used in the averaging process can be an important consideration when calculating the averaged coherence estimates as it involves a trade-off between resolution in the space and frequency domains. Frequency resolution is sharp for long space windows which yield smooth coherence curves. However, each averaged coherence estimate becomes an average over a larger number of Fourier coefficients. Altematively, shorter wavelength windows deteriorate the sharpness of the frequency resolution for coherence estimates. Given the length and sampling interval of our gridded surface and subsurface loads, we find, by trial and error, that our coherence estimates are well represented when averaged over 12 discrete wavebands (this yields the best agreement between the simulated and predicted coherence). As a rule of thumb, the first averaged coherence estimate should contain at least 5 Fourier coefficients. The coherence estimates shown here are obtained for bands equally spaced in the wavenumber domain.

\section{Simulation Results and Interpretation}

\section{Uncorrelated Loads}

Figure 1 shows the distribution of estimated $\widehat{T}_{e}$ and the respective coherence estimates for varying simulated $T_{0}$ values $\left(T_{\bullet}=20,40\right.$, and, $\left.80 \mathrm{~km}\right)$. The mean estimated effective elastic thickness $\left\langle\hat{T}_{e}\right\rangle$ and respective standard deviations are also shown for each study case. We note that the misfit between $T_{e}$ and $\left\langle\widehat{T}_{e}\right\rangle$, and thus the variance in our $\left\langle\widehat{T}_{e}\right\rangle$ estimates, is due to the finite extent of the fractal loads leading to a discrepancy between the ideal null correlation and the sampled $R$. An excellent agreement between $T_{0}$ and $\left\langle T_{\rangle}\right.$is found for $T_{0}=20 \mathrm{~km}\left(\left\langle\widehat{T}_{e}\right\rangle=21 \pm 2 \mathrm{~km}\right.$; Figure 1a). The mismatch between $T_{e}$ and $\left\langle\widehat{T}_{e}\right\rangle$ and respective standard deviations gradually increase as $\mathrm{T}_{0}$ increases (for $\mathrm{T}_{0}=\mathbf{4 0}$ and $80 \mathrm{~km},\langle\widehat{T}\rangle$ is $44 \pm 9 \mathrm{~km}$ and $94 \pm 29 \mathrm{~km}$, respectively). The coherence estimates shown in Figures 1d and 1e represent averages obtained for 100 statistically independent pairs of fractal surface and subsurface loads $(R=0)$. The open circles represent the simulated coherence averages obtained using (3). The predicted coherence estimates obtained using the simulated $\mathrm{T}_{\text {a }}$ and estimated $\left\langle\widehat{\mathrm{T}}_{\mathrm{e}}\right\rangle$ are shown as dotted and solid lines, respectively.

When short-wavelength loads are randomly placed on a thin elastic plate, the deflections of the corresponding compensating interfaces are not significant. As a consequence, the resulting topography and Bouguer gravity are not correlated. At long wavelengths, the correlation between topography and Bouguer gravity is perfect (coherence -1) as the rigidity of the plate cannot support the load. The wavenumber band at which the transition from high to low coherence values takes place is referred to as the diagnostic 


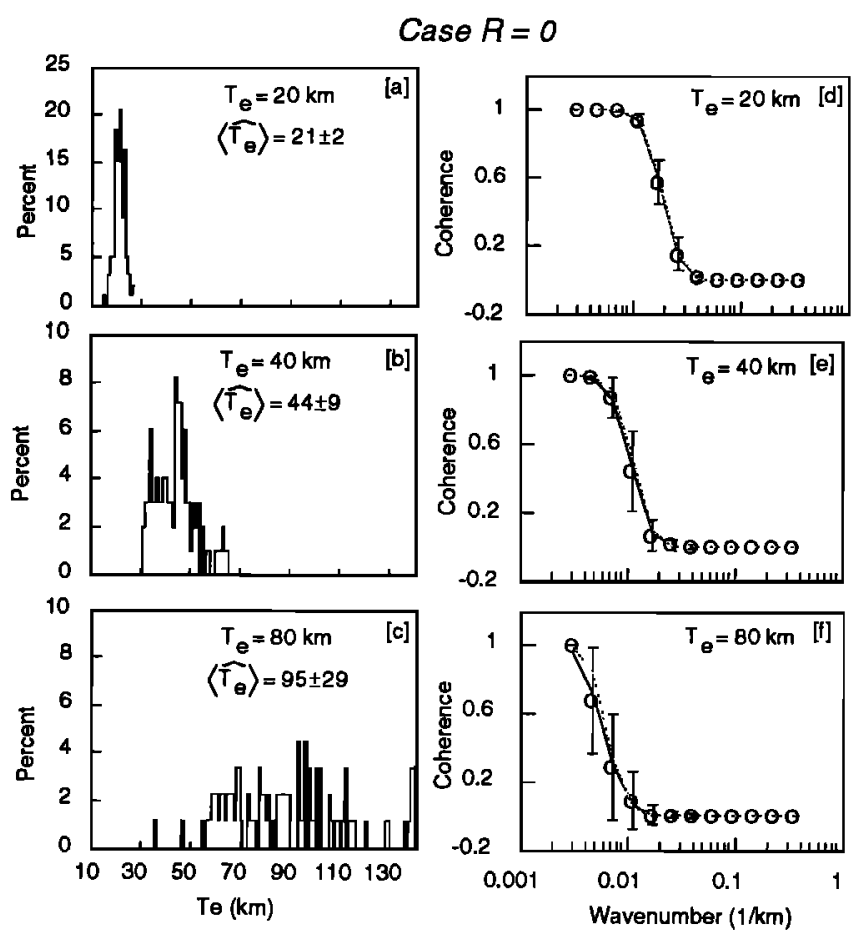

Figure 1. (a-c) Distributions of estimated $\widehat{T}_{c}$ and (d-f) corresponding coherence estimates for $T_{s}$ values of 20,40 , and $80 \mathrm{~km}$. The correlation between surface and subsurface loads is assumed to be null for all cases $(R=0)$. The dimensions of the gridded loads are $10^{\circ} \times 10^{\circ}$. The simulated coherence estimates are shown as open circles and the error bars denote 1 standard deviation. The predicted coherence estimates obtained using $\mathrm{T}_{\mathrm{c}}$ and $\left\langle\widehat{\mathrm{T}}_{\mathrm{e}}\right\rangle$ are shown as dotted and solid lines, respectively. Coherence estimates represent averages over 100 runs.

wavenumber band. Figures 1d and 1f clearly illustrate that as the plate gets more rigid, its ability to support longer wavelengths increases, and therefore the diagnostic wavenumber band gets shifted to longer wavelengths. Moreover, the range of $T_{c}$ values that fits the simulated coherence (for similar summed squared errors) increases, that is, the accuracy of the effective elastic thickness estimates degrades as $T_{e}$ increases.

The overestimate in $\widehat{T}_{e}$ for stiffer plates (for $T_{e}=80 \mathrm{~km}$, $\left\langle\widehat{T}_{e}\right\rangle$ is $94 \pm 24 \mathrm{~km}$; Figure 1c) is related to the physical dimensions of the grid used in this study $\left(\sim 10^{\circ} \times 10^{\circ}\right)$. For example, for an $80-\mathrm{km}$-thick plate, the characteristic flexural wavelength exceeds $1400 \mathrm{~km}$ (equation (2)), and thus the dimensions of the $\sim 10^{\circ} \times 10^{\circ}$ grid are not long enough to fully resolve the longest wavelengths within the diagnostic wavenumber band. In order to test this idea, we performed the same simulations using new grids twice the original grid size $\left(\sim 20^{\circ} \times 20^{\circ}\right)$. Figure $2 \mathrm{a}$ illustrates the better agreement between $\mathrm{T}_{\mathrm{e}}$ and $\left\langle\widehat{\mathrm{T}}_{\mathrm{e}}\right\rangle$ and a smaller standard deviation relative to Figure 1c. The fit between the simulated and predicted coherences has also significantly improved (Figure $2 \mathrm{~b}$ ).

Zuber et al. [1989], in their isostasy study of Australia, attempted to illustrate that the direct relationship between the best fitting $T_{c}$ and the size of the subregions used in the coherence analysis is not an artifact of the method (see their appendix). They proposed that by selecting two areas of equal dimensions from regions with distinct tectonic behavior, they get different rigidities. This is certainly true for the cases shown, but their best fitting $T_{e}$ for the more rigid area is not necessarily correct. According to our results, the accuracy of estimated $\widehat{T}_{e}$ is affected by the dimensions of the study area, in particular when the plate is fairly stiff (which seems to be the case for some provinces in Australia). The simulations presented in this study suggest that for an $80-\mathrm{km}$-thick plate, $\langle\widehat{T}\rangle$ may be overestimated by as much $18 \%$ from the true $\mathrm{T}_{\text {. }}$ under "ideal" conditions (null correlation between surface and subsurface loads). We predict that the mismatch between $T_{\text {。 }}$ and $\left\langle\widehat{T}_{e}\right\rangle$ gets progressively worse, that is, $\left\langle\widehat{T}_{e}\right\rangle$ is consistently overestimated as plate stiffness increases if the study area is not adequate to fully resolve the longest wavelengths. This might partially explain why flexural rigidity estimates for continental studies obtained using the coherence method $\left(D=10^{21}-10^{25} \mathrm{~N} \mathrm{~m}\right)$ are often higher than values obtained using conventional forward modeling techniques ( $\mathrm{D}=10^{23}-10^{24} \mathrm{~N} \mathrm{~m}$ ) [Walcott, 1970; Haxby et al., 1976; Karner and Watts, 1983; Royden and Karner, 1984; Royden and Burchfiel, 1989].

\section{Correlated Loads}

Figure 3 shows the distribution of estimated $\widehat{T}_{e}$ values obtained for 100 computer runs for two sets of correlation coefficient between surface and subsurface loads $(R=-0.2$ and
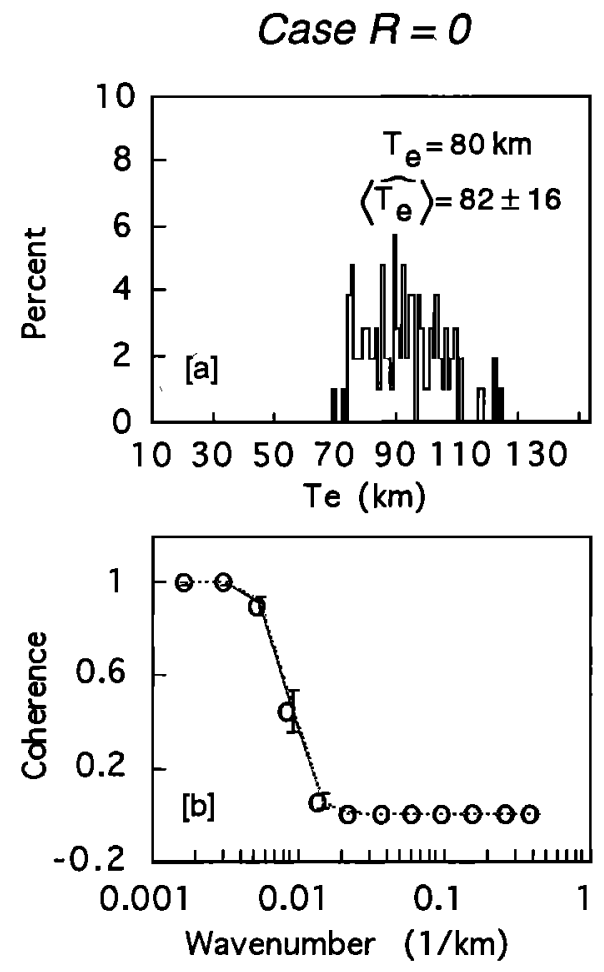

Flgure 2. Distributions of estimated $\widehat{T}_{e}$ and corresponding coherence estimates for stiff plates $\left(T_{0}=80 \mathrm{~km}\right)$. The correlation between surface and subsurface loads is assumed to be null $(R=0)$. The dimensions of the gridded loads are twice the one used in Figure $1\left(20^{\circ} \times 20^{\circ}\right)$. The better agreement between $T_{a}$ and mean $\left\langle\widehat{T}_{e}\right\rangle$ (in contrast to the stiff plate shown in Figure 1c) is related to the full resolution of the longest wavelengths. The simulated coherence estimates are shown as open circles and the error bars denote 1 standard deviation. The predicted coherence estimates obtained using $T_{0}$ and $\left\langle\widehat{T}_{e}\right\rangle$ are shown as dotted and solid lines, respectively. Coherence estimates represent averages over 100 runs. 

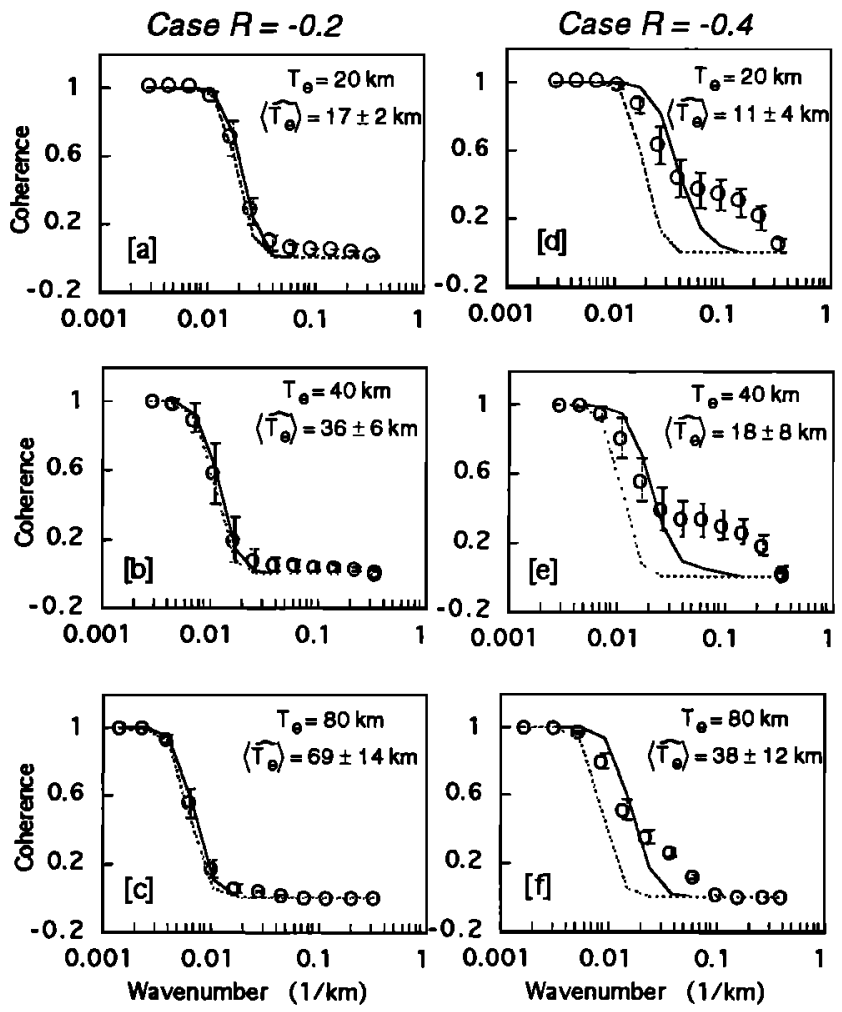

Figure 3. Distributions of estimated $\widehat{T_{e}}$ for the case in which the degree of correlation $R$ between surface and subsurface loads is (a-c) $R=-0.2$ and (d-f) $R=-0.6$. As $R$ increases, the estimated mean $\left\langle\widehat{T_{e}}\right\rangle$ (averaged for 100 runs) is progressively biased toward a low value relative to simulated $T_{0}$.

$\mathbf{R}=-\mathbf{0 . 6}$ ). The mean estimated $\left\langle\widehat{\left.\mathrm{T}_{e}\right\rangle}\right.$ and respective standard deviations for simulations with additional $R$ values are given in Table 2. For any given simulated $T_{0}$, as the correlation between surface and subsurface loads increases, the downward bias in $\left\langle\widehat{T}_{e}\right\rangle$ values gets progressively larger approaching a local loading scheme. The trend is clearly a decrease of $\left\langle T_{e}\right\rangle$ as $R$ increases.

The simulated coherence estimates obtained using (3) for the case of $R=-0.2$ and $R=-0.6$ are illustrated in Figure 4 . These represent averages obtained for the same set of computer simulations shown in Figure 3 . The predicted coherence estimates obtained using the simulated $T_{0}$ and estimated $\left\langle\widehat{T}_{a}\right\rangle$ (equation (7)) are shown as dotted and solid lines, respectively. For small correlations $(R=-0.2)$, the fit between the simulated and predicted coherence estimates is good at long to middle wavelengths. However, the fit at smaller wavelengths is not as good as for the case of null correlation, and it never drops completely to zero. When correlated loads are emplaced on a weak plate, the topography and gravity anomaly will automatically be correlated at most wavelengths. As a consequence, the diagnostic wavelength bands get shifted toward smaller wavelengths explaining the downward bias in $\widehat{T}_{e}$ (Figure 3 ). Clearly, as the degree of correlation increases, the misfit between simulated and predicted coherences gets progressively worse even at middle to long wavelengths. The characteristic shape of the coherence curve is no longer identifiable with increasing correlation between surface and subsurface loads.

It can be argued that the simulations we present in this study are not realistic in the sense that we apply a fixed amount of correlation $\mathbf{R}$ to the loads at all wavelengths. While it is certainly true that we could have used correlation schemes dependent on wavelength, it would be extremely difficult to test all the different possibilities as our coherence estimates would then also be tied to the band-limited cutoff value of $\mathbf{R}$. Although coherence values close to zero are usually expected at small wavelengths for realistic geological surfaces (in contrast to some of the simulations shown here), this part of the coherence curve does not play a major role when estimating the best fitting $T_{c}$. Even if the coherence estimates were "forced" to be close to zero at small wavelengths (for example, by introducing Gaussian noise in the gravity signal mimicking observational errors), our $\widehat{T}_{e}$ predictions would still be similar to the ones presented here if a correlation between surface and subsurface loads near the diagnostic waveband is assigned.

\section{Application to the Alps}

\section{Previous Studies}

The Alpine orogeny developed as a result of plate reorganizations in the Cretaceous time which led to a continent-continent collision between the Eurasian plate and the smaller Apulian plate [e.g., Coward and Dietrich, 1989]. Although there are significant differences in the existing models for the complex tectonic evolution of the Alps, we can approximately separate the events associated with the Alpine orogeny into two groups which account for the emplacement of large loads within and below the lithosphere. The first one, which started in the late Cretaceous/Paleogene times, involves a great deal of deformation of the downgoing Eurasian plate either by crustal [Mattauer, 1986] or lithospheric [Nicolas et al., 1990] wedging, depending on the favored interpretation for the location of the decoupling zone. The second event, during the late Paleogene/Neogene, led to successive emplacement of nappes, the external massifs and the folding of the previously undeformed foreland. By middle to late Miocene, crustal shortening across the eastern Alps was replaced by a dextral strike slip zone named Insubric line (see Figure 5a for location) [Royden and Burchfiel, 1989].

A considerable amount of uplift must have taken place in the Alps during the past $40 \mathrm{Ma}$ as suggested by the grade of metamorphism observed on exposed rocks. The maximum inferred uplift occurs to the north of the Insubric line where rocks were previously at depths of $20-25 \mathrm{~km}$ [Zingg et al., 1990]. The crustal structure beneath the Alps is fairly well constrained by recent deep reflection surveys, in particular across its western and central portions [Mueller et al., 1980; Meissner et al., 1987; Laubsher, 1990; Nicolas et al., 1990].

Table 2. Mean Estimated $<\widehat{T}_{e}>$ for Varying $T_{e}$ and $R$

\begin{tabular}{llll}
\hline$R$ & $T_{0}=20 \mathrm{~km}$ & $T_{0}=40 \mathrm{~km}$ & $\mathrm{~T}_{0}=80 \mathrm{~km}$ \\
\hline NULL & $21 \pm 2$ & $44 \pm 9$ & $95 \pm 29$ \\
-0.2 & $17 \pm 2$ & $36 \pm 6$ & $70 \pm 14$ \\
-0.4 & $13 \pm 2$ & $29 \pm 8$ & $55 \pm 11$ \\
-0.6 & $11 \pm 4$ & $18 \pm 8$ & $38 \pm 12$ \\
-0.8 & $2 \pm 2$ & $4 \pm 3$ & $19 \pm 8$
\end{tabular}



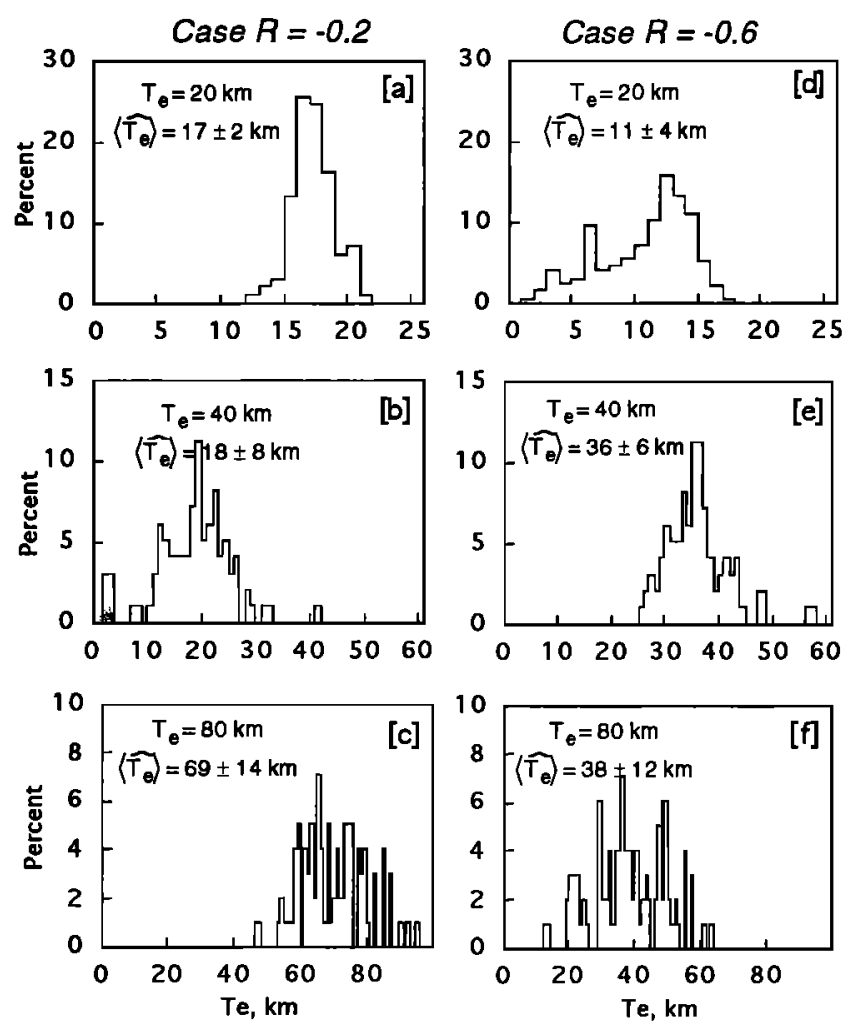

Figure 4. Coherence estimates (open circles) and corresponding standard deviations averaged over 100 runs for the cases shown in Figure 4 . The predicted coherence estimates obtained using $\mathrm{T}_{e}$ and $\left\langle\widehat{T}_{e}\right\rangle$ are shown as dotted and solid lines, respectively.

The average crustal thickness of $20-30 \mathrm{~km}$ beneath the Molasse foreland basin progressively changes to $40-60 \mathrm{~km}$ beneath the fold/thrust belt; Moho depths beneath the Po basin vary between 20 and $30 \mathrm{~km}$. The heat flow data across the western Alps follow a similar trend as Moho depths, that is, lower values beneath the maximum topographic relief $(\sim 63$ $\left.\mathrm{mW} / \mathrm{m}^{2}\right)$, progressively increase outward $\left(\sim 110 \mathrm{~mW} / \mathrm{m}^{2}\right)$ [Mueller et al., 1980] .

The Alps are probably the most extensively studied orogeny. Topography and gravity values are known at over 306,000 points. A minimum curvature algorithm applied iteratively from a coarse to a fine grid [Smith and Wessel, 1990] was used to generate the topography and Bouguer gravity grids shown as contoured maps in Figure 5 . The dimensions of the grids are $1122 \times 558 \mathrm{~km}$ and consists of 256 (longitude) $\times 128$ (latitude) points. The main feature in the gridded topographic map for the Alps is the fold/thrust belt system reaching a maximum elevation above sea level of $\sim 3000 \mathrm{~m}$. The largest amplitude gravity low observed in the Bouguer gravity maps for the Alps ( -200 mGal) lies approximately where the crust reaches its maximum thickness (40-60 km) [Meissner et al., 1987]. The narrow positive gravity anomaly on the concave part of the arcuate western Alps (Figure 5b) is associated with the presence of highdensity mafic/ultramafic bodies defining the Ivrea Zone [Mueller, 1982].

Like other thrust belts, the Alps are characterized by a positive-negative Bouguer gravity anomaly "couple". Based on a gravity study, Lyon-Caen and Molnar [1989] proposed that the Alps are not flexurally supported by the lithosphere but are dynamically supported by a relict subducted slab. Alternatively, Karner and Watts [1983] interpreted the positive component of the gravity anomaly "couple" as due to subsurface loads in the form of obducted crustal blocks and the negative component to the flexural deformation of the foreland basin. By invoking the presence of subsurface loads (assumptions on the mass and depth were constrained by the gravity signal), they performed a one-dimensional forward modeling of this positive-negative gravity anomaly "couple" observed in the Alps. According to their results, the flexural rigidity of the lithosphere changes by a factor of 2 across the orogenic belt, that is, $T_{a}$ values of $25 \mathrm{~km}$ for its highly arcuate western portion and $50 \mathrm{~km}$ for the more linear eastern portion.

Using foreland basin stratigraphy to constrain the basement configuration for the central Alps, Sinclair et al. [1991] obtained $T_{e}$ values in the range $5-15 \mathrm{~km}$, which are $\sim 10-20 \mathrm{~km}$ less than the $T_{c}$ estimate proposed by Karner and Watts [1983] for the western Alps. In the gravity studies mentioned here, the two dimensionality assumed when performing a one-dimensional modeling across highly arcuate orogenic belts is questionable. Watts and Cochran [1974] showed that the amplitude of deflection computed assuming a two-dimensional square load may be as much as 4 times less than the one predicted by one-dimensional loads. In this paper we reassess $T_{0}$ estimates in the Alps using a two-dimensional coherence analysis in which no a priori assumptions on the mass and depth of the subsurface loads are made.

\section{Coherence Study}

Figure 6a illustrates the observed coherence values (open circles) and respective error bars obtained by Fourier transformation of the Bouguer gravity and topography data shown in Figure 5. The predicted coherence curve (solid line) for the best fitting $T_{0}$ assumes a single subsurface interface (Moho) at a depth of $30 \mathrm{~km}$ which was obtained by downward continuation of the Bouguer gravity. After solving for the load components for an initial guess of $T_{e}$, the predicted coherence was calculated using (7). The best fitting $T_{e}$ for the entire Alps, defined as the one that yields the smallest misfit between observed and predicted coherence, is $-27 \mathrm{~km}$. The $T_{\text {o }}$ range for which the predicted coherence curves fall within 1 standard deviation for at least $2 / 3$ of the coherence estimates within the transitional wave bands is $23-42 \mathrm{~km}$.

In order to evaluate whether the Alpine fold/thrust belt consists of provinces of different rigidities, we divide the gridded data into two different subsets: the highly arcuate (radius of curvature is $-251 \mathrm{~km}$ to the west of $9^{\circ} \mathrm{E}$; Figure $6 \mathrm{~b}$ ) and the more linear segment (radius of curvature is $\sim 501 \mathrm{~km}$ to the east of $9^{\circ} \mathrm{E}$; Figure $6 \mathrm{c}$ ). According to our results, the elastic thickness of the continental lithosphere for the arcuate western portion of the Alps ( $T_{0} \sim 31 \mathrm{~km}$; range $30-39 \mathrm{~km}$ ) does not differ significantly from estimates obtained for the linear eastern portion $\left(T_{c}-35 \mathrm{~km}\right.$; range $\left.33-40 \mathrm{~km}\right)$. The slightly larger $T_{e}$ estimates for west and east Alps relative to value obtained for the entire Alps is not statistically significant.

The correlation coefficients between the initial surface and subsurface loads (inverse loads) obtained for the best fitting $T_{\text {}}$ values for western and eastern Alps are shown in Figure 7. The inverse loads were calculated using Forsyth [1985] formulations. No significant correlation between surface and subsurface loads is found for either the western or the eastern Alps. Assuming a maximum linear correlation coefficient 

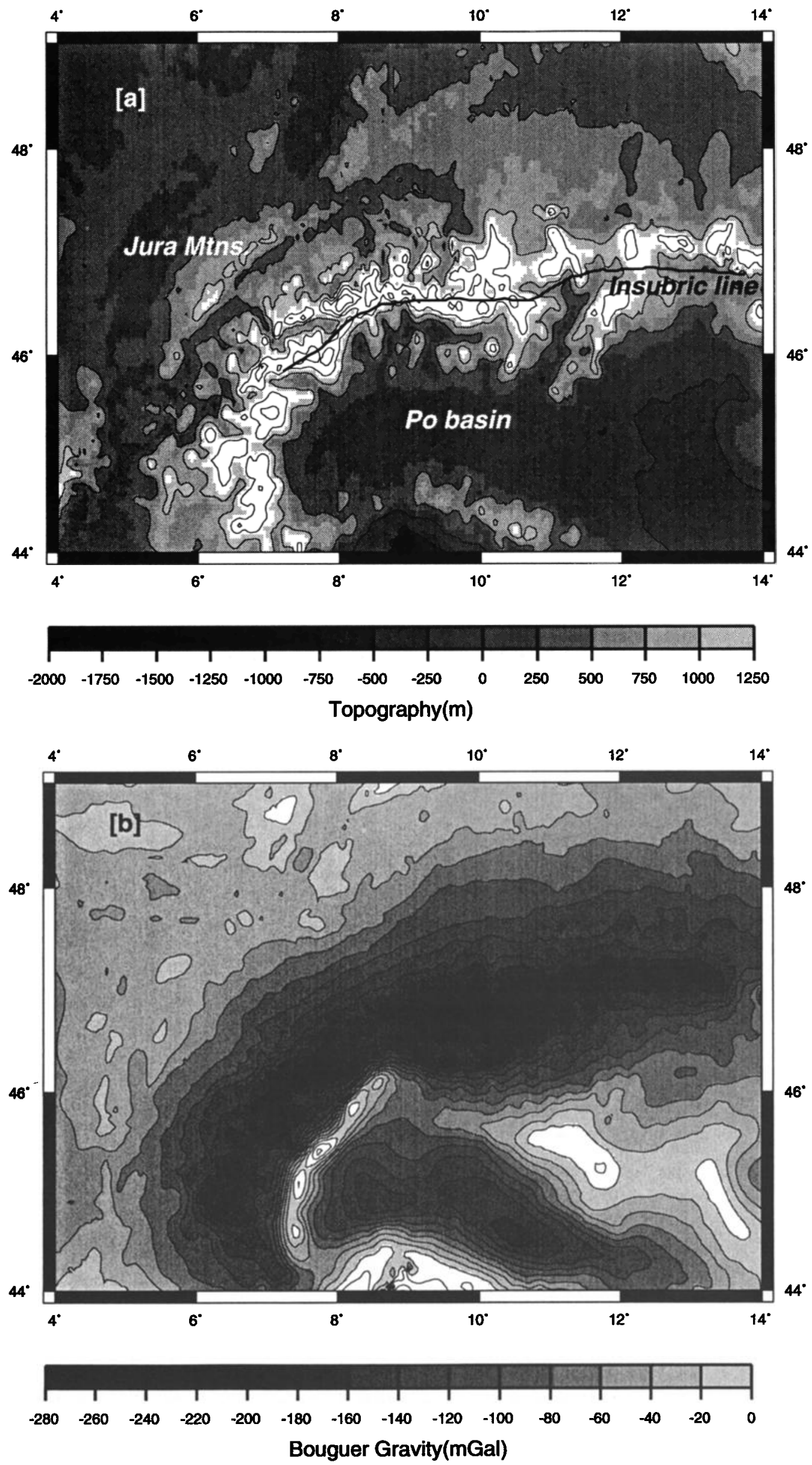

Figure 5. (a)Topography and (b) Bouguer gravity maps for the Alps and adjacent areas contoured at $500 \mathrm{~m}$ and $20 \mathrm{mGal}$ intervals, respectively. The grids used to generate these maps have a grid space of 3 arc $\mathrm{min}$. 

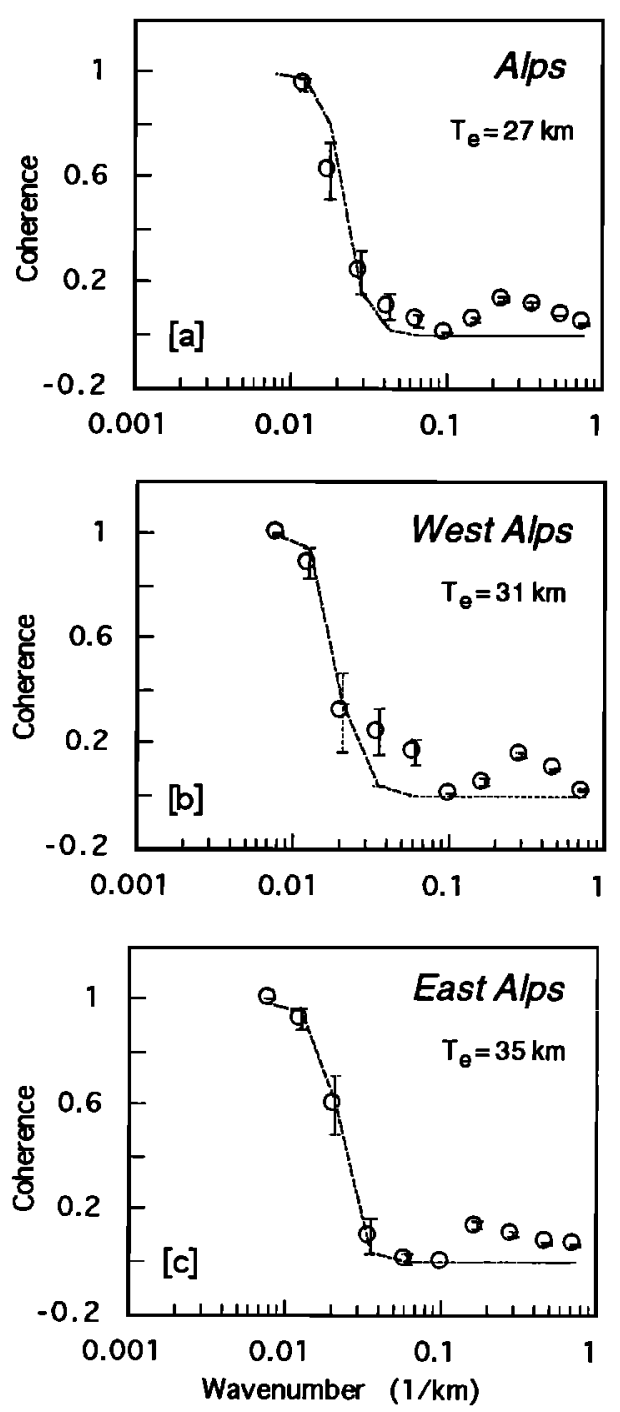

Figure 6. Observed (open circles) and predicted (solid line) coherence estimates for (a) the entire fold/thrust Alpine system, (b) the highly arcuate western portion of the Alps, and (c) the linear eastem portion. Error bars denote 1 standard deviation using Bendat and Piersol [1980] formulation. The best fitting $T_{0}$ is the value that minimizes the misfit between observed and predicted coherences. A subsurface interface (Moho) at a depth of $30 \mathrm{~km}$ together with the parameters shown in Table 1 were assumed in the $T_{0}$ inversion .

between initial surface and subsurface loads of -0.4 at long wavelengths (approximately the topmost value for the longest wavelength in Figure 8a), the permissible range of $T_{a}$ estimates is $23-55 \mathrm{~km}\left(\mathrm{D}=7.0 \times 10^{22}-9.6 \times 10^{23} \mathrm{~N} \mathrm{~m}\right)$ (using the Monte Carlo $\left\langle\widehat{T}_{e}\right\rangle$ estimated for a $T_{b}$ of $40 \mathrm{~km}$ and linear correlation coefficient $R=-0.4$; see Table 2).

Based on a compilation study of 15 mountain belts (including Karner and Watts [1983] results), McNutt et al. [1988] proposed a positive correlation between $T_{0}$ values and radius of curvature. The lack of significant differences between our best fitting $T_{\text {}}$ results for the western and eastern Alps does not lend further support to this hypothesis. It is important to point out that most of $\mathrm{T}_{0}$ estimates used in the McNutt et al. [1988] compilation study were derived from one-dimensional modeling which, as stated before, might be proned to errors for arcuate mountain belts. Although it is intuitive to think that thinner plates are easier to bend than thicker ones, other factors may also affect the curvature of mountain belts in plan view. We suggest that plate processes combined with the precollisional geometry of the passive margins offer the simplest explanation for the observed difference in shape between the westem and eastern Alps. The arcuate shape of the western Alps is probably related to the Paleogene to Recent convergent trend, whereas the linear shape of the eastern Alps is most likely related to the dextral strike-slip motion that replaced convergence in the Miocene.

The thickness of the lithosphere obtained from gravity studies together with estimates on the time of loading have often been used to infer the depth of the controlling isotherm and the thickness of the thermal plate [e.g., Karner and Watts, 1983; McNutt et al., 1988]. As pointed out by McNutt et al. [1988], $T_{0}$ estimates yield a lower bound on the thermal plate thickness because most of the processes acting on the lithosphere will always tend to make it less rigid. In the Alps, for example, there is significant evidence from K-Ar dating on different groups of mineral associations in the Lepontine (central Alps) and Tauern (eastern Alps) regions for a peak metamorphic phase related with high temperatures at $\sim 15-30$ Ma [Ernst, 1973; Frey et al., 1974]. More recently, it has been also suggested that heat advection by upward-migrating fluids may represent an effective way of raising temperatures in the lower crust [Hoish, 1991]. Given the uncertainties related to the permissible range of $T_{0}$ estimates and the thermal age of the lithosphere, we believe that the thickness of the thermal plate and/or the controlling isotherm for the Alps cannot be objectively constrained.

\section{Summary and Conclusions}

This study provided important insights on the accuracy of $T_{0}$ estimates obtained using the coherence method. By running simple Monte Carlo computer simulations we were able to
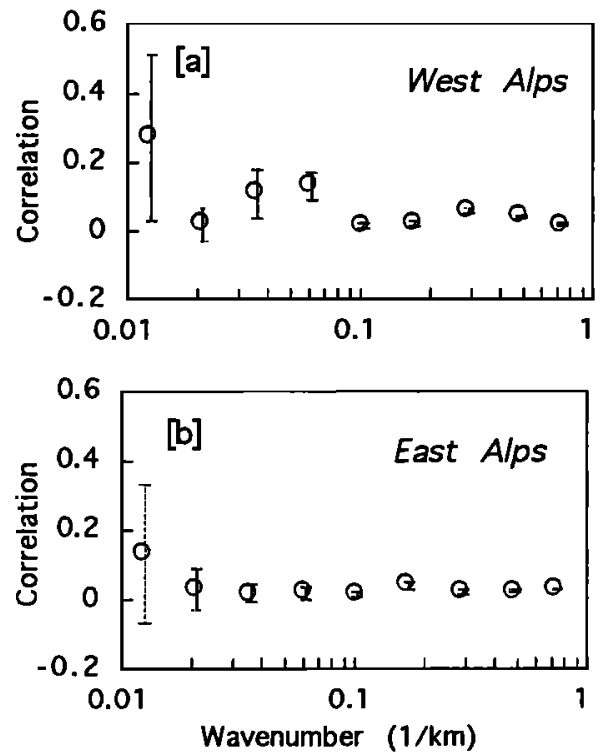

Figure 7. Correlation coefficients between the amplitude of the initial surface and subsurface inverse loads for (a) west and (b) east Alps. The inverse loads were obtained assuming a Moho depth of $30 \mathrm{~km}$, the parameters in Table 1 and best fitting $T_{\text {s }}$ shown in Figure 6. 
quantify the bias and variance in $T_{0}$ estimates when surface and subsurface loads are statistically correlated. The main conclusions can be summarized as follows:

1. For the case when there is no statistical correlation between surface and subsurface loads $(R=0)$, the mean estimated $\left\langle\widehat{T}_{e}\right\rangle$ values obtained using the coherence method are in excellent agreement with the computer simulated $\mathbf{T}_{\boldsymbol{e}}$.

2. The accuracy of $\langle\widehat{T}\rangle$ estimates decreases as the plate thickness increases.

3. The ratio of flexural wavelength to the dimensions of the study area place an important constraint in the accuracy of $\left\langle\widehat{T_{e}}\right\rangle$ values. Upwardly biased and poorly resolved $\left\langle\widehat{T}_{e}\right\rangle$ estimates are obtained when the dimensions of the study area are not adequate to fully resolve the longest wavelengths. This may explain why $T_{\text {, }}$ values obtained using the coherence method are often higher than $T_{\text {e }}$ estimates obtained using forward modeling techniques.

4. As the degree of correlation increases we observe a clear downward bias in $\langle\widehat{T}\rangle$ values. When surface and subsurface loads are correlated by only a small amount (e.g., $R=-0.2$ ), the fit between predicted and simulated coherence is still good for the longest wavelengths within the diagnostic wavebands; at smaller wavelengths the simulated coherence will always be higher than the predicted ones.

In the second part of this study we used the coherence method to reassess $T_{\text {}}$ estimates for the Alps in light of the results/limitations discussed above. The main conclusions are as follows:

1. The best fitting $T_{e}$ value for the entire Alps is $\sim 27 \mathrm{~km}$ (range $23-42 \mathrm{~km}$ ).

2. The similarity between our estimated $T_{0}$ results for the western ( $\sim 31 \mathrm{~km}$; range $30-39 \mathrm{~km})$ and eastern Alps ( $\sim 35 \mathrm{~km}$; range $33-40 \mathrm{~km}$ ) obtained using the coherence method does not support the hypothesis that $T_{e}$ and horizontal radius of curvature of mountain belts are correlated. In the worst possible scenario of $40 \%$ correlation at long wavelengths between initial surface and subsurface loads $(R=-0.4)$, the estimated $T_{e}$ for west and east Alps may vary in the range of 23-55 km as predicted by the Monte Carlo simulations in the first part of this study. We suggest that the observed difference in the shape of the westem and eastern Alps can be most simply explained in terms of plate processes combined with the precollisional geometry of the passive margins.

3. Given the combined uncertainties in $T_{e}$ and thermal age estimates, we cannot constrain the thermal thickness of the lithosphere and/or the controlling isotherm for the Alps.

\section{Appendix : Degree of Correlation and Pearson's Linear Correlation Coefficient}

Consider two uncorrelated zero-mean surfaces $s_{1}$ and $s_{2}$ (function of $x, y$ ). If $E\{$.$\} is the expectation operator, then the$ two surfaces are uncorrelated when [Papoulis, 1984]

$$
E\left\{s_{1} s_{2}\right\}=0
$$

Suppose, also, that both $s_{1}$ and $s_{2}$ have a variance $\sigma^{2}$ :

$$
E\left\{s_{1}^{2}\right\}=E\left[s_{2}^{2}\right\}=\sigma^{2}
$$

Consider now a third surface $s_{3}$ related to $s_{1}$ and $s_{2}$ by $R$ $(-1<R<0)$ :

$$
s_{3}=R s_{1}+\sqrt{1-R^{2}} s_{2}
$$

The Pearson's linear correlation coefficient $\rho$ is defined for the surfaces $s_{1}$ and $s_{3}$ as

$$
p=\frac{E\left\{s_{12} s_{3}\right\}}{\sqrt{E\left[s_{1}{ }^{2}\right] E\left[s_{3}{ }^{2}\right\}}}
$$

We have

$E\left\{s_{1} s_{3}\right\}=E\left\{s_{1}\left[R s_{1}+\sqrt{1-R^{2}} s_{2}\right]\right\}=E\left\{R s_{1}{ }^{2}\right\}=R \sigma^{2}$

because $E\left\{s_{1} s_{2}\right\}=0$ (equation (A1)). It also follows that

$$
\begin{aligned}
E\left[s_{3}{ }^{2}\right. & =E\left[\left[R s_{1}+\sqrt{1-R^{2}} s_{2}{ }^{2}\right]\right. \\
& =E\left[R^{2} s_{1}{ }^{2}\right]+E\left[\left(1-R^{2}\right) s_{2}{ }^{2}\right\} \\
& =\left(R^{2}+1-R^{2}\right) \sigma^{2}=\sigma^{2}
\end{aligned}
$$

Substituting (A5) and (A6) in (A4), we obtain

$$
\rho=\frac{R \sigma^{2}}{\sqrt{\sigma^{4}}}=R
$$

where $\mathbf{R}$ is Pearson's correlation coefficient. If we rescale $s_{1}$ and $s_{3}$ by constant multiplicative factors $\alpha_{1}$ and $\alpha_{3}$, that is, define

$$
\tilde{s}_{1}=\alpha_{1} s_{1}, \tilde{s}_{3}=\alpha_{3} s_{3}
$$

the result is unchanged:

$$
\tilde{\rho}=\frac{E\left\{\tilde{s}_{1} \tilde{s}_{3}\right]}{\sqrt{E\left\{\tilde{s}_{1}{ }^{2}\right\} E\left[\tilde{s}_{3}{ }^{2}\right\}}}=\frac{\alpha_{1} \alpha_{3} E\left[s_{1} s_{3}\right\}}{\alpha_{1} \alpha_{3} \sqrt{E\left[s_{1}{ }^{2}\right] E\left[s_{3}{ }^{2}\right\}}}=p
$$

Acknowledgments. Ana Macario benefited from discussions with Garry Kamer and Jeffrey Weissel and is grateful to their support in the first stages of this research. Donna Blackman and two anonymous reviewers had several comments that improved the manuscript. Lamont-Doherty Earth Observatory contribution $\mathbf{5 3 5 2 .}$

\section{References}

Banks, R. J. and C. J. Swain, The isostatic compensation of East Africa, Proc. R. Soc. London. A, 364, 331-352, 1978.

Banks, R. J., R. L. Parker, and S. P. Huestis, Isostatic compensation on a continental scale: Local vs. regional mechanisms, Geophys. J. $R$. Astron.. Soc., 51, 431-452, 1977.

Bechtel, T. D., D. W. Forsyth, and C. J. Swain, Mechanisms of isostatic compensation in vicinity of the East Africa Rift, Kenya, Geophys. J. R. Astron. Soc., 90, 445-465, 1987.

Bechtel, T. D., D. W. Forsyth, V. L. Shartpon, and R. A. F. Grieve, Variations in effective elastic thickness of the North America lithosphere, Nature, 343, 636-638, 1990.

Bendat, J. S. and A. G. Piersol, Engineering Applications of Correlation and Spectral Analysis, John Wiley, New York, 1980.

Blackman, D. K, and D. W. Forsyth, Isostatic compensation of tectonic features of the Mid-Atlantic Rdge: 25-27930'S, J. Geophys. Res., 96, 11741-11758, 1991.

Coward, M., and D. Dietrich, Alpine tectonics - An overview, in Alpine Tectonics, edited by M.P. Coward, D. Dietrich and R.G. Park, Geol. Soc. Spec. Publ. London, 45, 1-29, 1989.

Dewey, J. F., W. C. Pitman III, W. B. F. Ryan and J. Bonnin, Plate tectonics and the evolution of the Alpine system, Geol. Soc. Am. Bull., 84, 3137-3180, 1973. 
Doman, L. M., and B. T. R. Lewis, Experimental isostasy, 1, Theory of the determination of the Earth's isostatic response to a concentrated load, J. Geophys. Res., 75, 3357-3365, 1970.

Ebinger, C. J., T. D. Bechtel, D. W. Forsyth, and C. O. Bowin, Effective elastic thickness beneath the East-African and Afar domes, $J$. Geophys. Res., 94, 9353-9371, 1989.

Emst, W. G., Interpretative synthesis of the metamorphism in the Alps, Geol. Soc. Am. Bull., v 84, 2053-2078, 1973.

Forsyth, D. W., Subsurface loading and estimates of the flexural rigidity of continental lithosphere, J. Geophys. Res., 90, 12623-12632, 1985.

Frey, M., J. C. Hunziker, W. Frank, J. Bocquet, G. V. D. Piaz, E. Jager and E. Niggli, Alpine metamorphism - A review, Schweiz. Mineral. Petrogr. Mitt., 54, 247-277, 1974.

Haxby, W. F., D. L. Turcotte, and J. M. Bird, Thermal and mechanical evolution of the Michigan basin, Tectonophysics, 36, 57-75, 1976.

Hoish, T. D., The thermal effects of pervasive and channelized fluid flow in the deep crust, J. Geol., 99, 69-90, 1991.

Kamer, G. D. and A. B. Watts, Gravity anomalies and flexure of the lithosphere at mountain ranges, J. Geophys. Res., 88, 10449-10477, 1983.

Laubscher, H., The problem of the Moho in the Alps, Tectonophysics, $182,9-20,1990$.

Lewis, B. T. R., and L. M. Dorman, Experimental isostasy, 2, An isostatic model for the United States derived from gravity and topography data, J. Geophys. Res., 75, 3371-3386, 1970.

Louden, K. E., A comparison of isostatic response of bathymetric features in the North Pacific Ocean and the Philippine Sea, Geophys. J. R. Astron. Soc., 64, 393-424, 1981.

Lyon-Caen, H., and P. Molnar, Constraints on the deep structure and dynamic processes beneath the Alps and adjacent regions from an analysis of gravity anomalies, Geophys. J. Int., 99. 19-32, 1989.

Mandelbrot, B., The Fractal Geometry of Nature, 468 pp., W. H. Freeman, New York, 1982.

Mark, D. M., and B. P. Aroson, Scale-dependent fractal dimensions of topographic surfaces: An empirical investigation with applications in geomorphology and computer mapping, Math. Geol., 16, 671-683, 1984.

Mattauer, M., Intracontinental subduction, crust-mantle decollement and crustal stacking wedge in the Himalayas and other collision belts, Geol. Soc. Spec. Publ., London, 19, 37-50, 1986.

McKenzie, D. P., and C. Bowin, The relationship between bathymetry and gravity in the Atlantic Ocean, J. Geophys. Res., 81, 1903-1915, 1976.

McNutt, M. K., Implications of regional gravity for the state of stress in the Earth's crust and upper mantle, J. Geophys. Res., 85, 6377-6396, 1980.

McNutt, M. K., and H. W. Menard, Constraints on the yiled strength in the oceanic lithosphere derived from observations of flexure, $J$. Geophys. Res., 87, 363-394, 1982.

McNutt, M. K., Influence of plate subduction on isostatic compensation in northem Califomia, Tectonics, 2, 399-415, 1983.

McNutt, M. K., M. Diament and M. G. Kogan, Variations of elastic plate thickness at continental thurst belts, J. Geophys. Res., 93, 88258838, 1988.

McNutt, M. K., and R. L. Parker, Isostasy in Australia and the evolution of the compensation mechanism, Science, 199, 773-775, 1978.

Meissner, R., T. Wever, and E. R. Fluch, The Moho in Europe Implications for crustal development, Ann. Geophys., 5B(4), 357364, 1987.

Mueller, S., Deep structure and recent dynamic in the Alps, in Mountain Building Processes, edited by K. J. Hsu pp. 181-193, Academic, San Diego, Calif., 1982.

Mueller, S., J. Ansorge, R. Egloff, and E. Kissling, A crustal cross section along the Swiss Geotraverse from the Rhinegraben to the Po Plain, Eclogae Geol. Helv., 73, 463-483, 1980.

Munk, W. H., and D. E. Cartwright, Tidal spectroscopy and prediction: Philos. Trans. Soc. London, 31, 447-455, 1966.

Nicolas, A., A. Him, R. Nicolich, R. Polino, and ECORS-CROP Working Group, Lithospheric wedging in the westem Alps inferred from the ECORS-CROP, Geology, 18, 587-590, 1990.

Papoulis, A., Probability, Random Variables and Stochastic Processes, 2nd ed., 576 pp., McGraw-Hill, New York, 1984.

Parker, R. L., The rapid calculation of potential anomalies, Geophys. J. R. Astron. Soc., 31, 447-455, 1972.

Peitgen, H. O., D. Saupe (Eds), The Science of Fractal Images, 312 pp., Springer-Verlag, New York, 1988.

Press, W. H., B. P. Flannery, S. A. Teukolsky, and W. T. Vetterling, Numerical Recipes in C: The Art of Scientific Computing, 735 pp., Cambridge University Press, New York, 1991.

Ribe, N. M., and A. B. Watts, The distribution of intraplate volcanism in the Pacific Ocean basin: A spectral approach, Geophys. J. R. Astron. Soc., 71, 331-362, 1983.

Royden, L. H., and G. D. Kamer, Flexure of the continental lithosphere beneath the Apennines and Carpathians: Evidence for an insufficient topographic load, AAPG Bull., 68, 704-712, 1984.

Royden, L., and B. C. Burchfiel, Are systematic variations in thrust belt style related to plate boundary processes? (The westem Alps versus Carpathians), Tectonics, 8, 51-61, 1989.

Sinclair, H. D., B. J. Coakley, P. A. Allen, and A. B. Watts, Simulation of the foreland basin and stratigraphy using a diffusion model of mountain belt uplift and erosion: An example from the central Alps, Switzerland, Tectonics, 10, 599-620, 1991.

Smith, W. H. F., and P. Wessel, Gridding with continuous curvature splines in tension, Geophysics, 55, 293-305, 1990.

Turcotte, D. L., and G. Schubert, Geodynamics: Applications of Continuum Physics to Geological Problems, 450 pp., John Wiley, New York, 1982.

Walcott, R.I., Flexural rigidity, thickness and viscosity of the lithosphere, J. Geophys. Res., 75, 3941-3954, 1970.

Watts, A. B., and J. R. Cochran, Gravity anomalies and flexure of the lithosphere along the Hawaiian-Emperor seamount trench, Geophys. J. R. Astron. Soc., 38, 119-141, 1974.

Watts, A. B., and M. Talwani, Gravity anomalies seaward of deep-sea trenches and their tectonic implications, Geophys. J. R. Astron. Soc., $36,57-90,1974$.

Watts, A. B., J. H. Bodine, and N. R. Ribe, Observations of flexure and geologic evolution of the Pacific Ocean Basin, Nature, 238, 532-537, 1980.

Watts, A. B., U. S. ten Brick, P. Buhl, and T. D. Brocker, A multichannel seismic study of the lithosphere flexure across the HawaiianEmperor seamount chain, Nature, 315, 105-111, 1985.

Zingg, A., M. R. Handy, J. C. Hunzinker, and S. M. Schmid, Tectonometamorphic history of the Ivrea Zone and its relationship to the crustal evolution of the Southem Alps, Tectonophysics, 182, 169 $192,1990$.

Zuber, M. T., T. D. Bechtel, and D. W. Forsyth, Effective elastic thickness of the lithosphere and the mechansms of isostatic compensation in Australia, J. Geophys. Res., 94, 9353-9366, 1989.

A. Macario and W. F. Haxby, Lamont-Doherny Earth Observatory, P. O. Box 1000, Route 9W, Palisades, NY 10964-8000. (e-mail: ana@lamont.ldeo.columbia.edu; bill@lamont. ldeo.columbia.edu)

A. Malinvemo, Schlumberger-Doll Research, Old Quarry Road, Ridgefield, CT 06877. (e-mail: alberto@com.slb.sdr)

(Received March 14, 1994; revised March 20, 1995;

accepted March 22, 1995.) 